\title{
FATORES ASSOCIADOS AO BAIXO PESO AO NASCER EM MUNICÍPIO DO NORTE DO BRASIL
}

\section{FACTORS ASSOCIATED WITH THE LOW BIRTH WEIGHT IN MUNICIPALITY IN NORTHERN BRAZIL}

\author{
Raquel da Rocha Paiva Maia ${ }^{1}$ \\ José Maria Pacheco de Souza ${ }^{2}$
}

Maia RRP; Souza JMP. Fatores associados ao baixo peso ao nascer em Município do Norte do Brasil. Rev. Bras. Cresc. e Desenv. Hum. 2010; 20(3) 735-744.

\section{Resumo}

Introdução: o baixo peso ao nascer (BPN) é considerado um dos mais importantes problemas de saúde pública em todo o mundo, contribuindo, substancialmente, para a morbimortalidade infantil. Objetivos: estimar a proporção de baixo peso ao nascer e identificar os fatores associados. Método: estudo transversal onde se analisaram 3220 declarações de nascidos vivos referentes aos partos ocorridos no município de Cruzeiro do Sul, Estado do Acre, no período de 2006 e 2007, de mães residentes nesta localidade. Na análise, utilizouse regressão linear generalizada família Poisson ligação logarítmica com variância robusta, simples e múltipla. Adotou-se nível de significância de 0,10 . Resultados: a proporção de baixo peso ao nascer foi $9,13 \%$. Os fatores associados ao baixo peso ao nascer foram: prematuridade; nascimento no domicílio; sexo feminino; idades maternas entre 12 e 13 anos, 16 e 17 anos, 18 e 19 anos, 35 e mais anos; realização de 1 a 3 consultas de pré-natal, crianças não brancas, mães sem ocupação fora do lar e mães solteiras. Conclusão: são poucos (ou nenhum) os fatores suscetíveis de mudança ou controle com ações isoladas de saúde. Estratégias de ampla abrangência são necessárias para a redução da proporção de baixo peso ao nascer em Cruzeiro do Sul, Acre e, uma vez ocorrido baixo peso ao nascer, atenção especial deve ser proporcionada à criança.

Palavras-chave: baixo peso ao nascer; nascidos vivos; sistemas de informação.

1 Mestre em Saúde Pública pela Faculdade de Saúde Pública/USP. Professora do curso de graduação em Enfermagem da Universidade Federal do Acre.

Correspondência para: raquelmaia@usp.br

2 Doutor em Saúde Pública pela Faculdade de Saúde Pública/USP.. Professor Titular (aposentado) da Universidade de São Paulo. Av. Dr. Arnaldo, n 715 CEP: 01246-904 - S. Paulo/SP - Brasil. Fones (11)3061-7747; (11)3768-8612;(11)3714-2403. Email: www.fsp.usp.br

Artigo resultante de dissertação: MAIA, R. R. P. Fatores associados ao baixo peso ao nascer no Município de Cruzeiro do Sul, Acre [dissertação de mestrado]. São Paulo: Faculdade de Saúde Pública da USP; 2009, junto ao Departamento de Epidemiologia da Faculdade de Saúde Pública/USP 


\begin{abstract}
Introduction: the low birth weight (LBW) is considered one of the most important public health problems around the world, contributing substantially to infantile morbidity and mortality. Objectives: to estimate the proportion of low birth weight and identify the factors associated. Method: cross-sectional study which examined 3220 statements of births relating to births occurring in the city of Cruzeiro do Sul, Acre, in the period 2006 to 2007, of resident mothers in this locality. In the analysis, linear regression generalized with Poisson family logarithmic linking robust variance, simple and multiple was used, with a significance level of 0.10 . Results: the proportion of low birth weight was $9.13 \%$. Factors associated with low birth weight were: prematurity, birth at home, female, maternal age between 12 and 13 years, 16 and 17 years, 18 and 19 years, more than 34 years, achieving 1-3 pre-natal consultations, non-white children, mothers with no occupation outside home and single mothers. Conclusion: few (or none) factors found are susceptible to change or control with isolated health actions. Wide variety of strategies are needed to reduce the proportion of LBW in the city of Cruzeiro do Sul, Acre, and when low birth weight occurred attention should be given to the child.
\end{abstract}

Key words: low birth weight; live birth; information systems.

\section{INTRODUÇÃO}

As consequências do baixo peso ao nascer (BPN) e suas repercussões o caracterizam como um importante problema de saúde pública. Estudos revelam alguns dos problemas associados ao BPN: maior mortalidade infantil, risco aumentado de morrer prematuramente de doença cardiovascular, hipertensão e diabetes, na idade adulta e maior morbidade como comprometimento na estatura e déficit nutricional ${ }^{1-4}$.

O BPN é definido como o peso inferior a $2500 \mathrm{~g}^{5}$. Seus determinantes incluem a prematuridade e a restrição de crescimento intrauterino (RCIU), ou uma combinação de ambos. A alta proporção de baixo peso ao nascer, encontrada nos países em desenvolvimento, tem sido associada principalmente a bebês com RCIU, enquanto a prematuridade constitui o elemento principal nos países desenvolvidos ${ }^{6}$. Algumas causas de partos prematuros incluem: infecções genitais, partos múltiplos, hipertensão materna, disfunções uterinas, trabalho materno pesado, assistência pré-natal inadequada e baixa idade materna ${ }^{7-10}$. Entre os fatores associados à RCIU estão medidas antropométricas maternas desfavoráveis (baixa estatura, baixo peso pré-gestacional e reduzido índice de massa corporal), álcool e fumo durante a gestação e infecções fetais?

O BPN está presente em $15,5 \%$ de todos os nascimentos ocorridos no mundo. No entanto, o problema não ocorre de maneira uniforme entre as diversas localidades, mas está relacionado à situação socioeconômica que as mesmas apresentam. A maior porcentagem de crianças com esse agravo concentra-se em duas regiões do mundo, Ásia e África, com respectivamente $27 \%$ e $22 \%$ de todos os nascidos vivos com baixo peso nos países em desenvolvimento ${ }^{11}$. Em países desenvolvidos, de modo geral a proporção de BPN situa-se entre 4\% e $6 \%{ }^{12}$. Em 2005, o Brasil apresentou uma proporção de $8,1 \%$ e o Estado do Acre de $6,9 \%{ }^{13}$.

No Brasil, existem os Sistemas de Informações em Saúde, que coletam dados importantes para a construção de diagnósticos e avaliação das ações de saúde. O Ministério da Saúde implantou, em 1990, o Sistema de In- 
formações sobre Nascidos Vivos (SINASC), que utiliza como fonte de dados um documento padronizado em todo o país, a Declaração de Nascido Vivo (DN). O preenchimento da DN é obrigatório para todos os nascidos vivos no Brasil. A declaração coleta informações sobre a gestante, o parto e o recém-nascido, permitindo caracterizar as condições de nascimento e facilitando, dessa forma, a detecção de falhas e o direcionamento das ações na área materno-infantil ${ }^{14}$.

Além disso, possibilita a realização de investigação sobre os fatores que contribuem de forma mais acentuada para o nascimento de crianças com baixo peso. Assim, os objetivos são estimar a proporção de baixo peso ao nascer e verificar os fatores associados ao baixo peso em nascidos vivos.

\section{MÉTODO}

Estudo transversal que utilizou como fonte de dados a Declaração de Nascido Vivo. A população de estudo incluiu os nascidos vivos de partos ocorridos no município de Cruzeiro do Sul, Estado do Acre, no período de 2006 e 2007, de mães residentes nesta localidade.

As variáveis do estudo foram: peso ao nascer (baixo peso ao nascer: $<2500 \mathrm{~g}$; peso normal: e" $2500 \mathrm{~g}$ ); idade materna (12 e 13; 14 e $15 ; 16$ e $17 ; 18$ e $19 ; 20$ a $24 ; 25$ a $34 ; 35$ ou mais anos; ignorada/não preenchida); estado civil (solteira; outros: casada, viúva ou separada judicialmente/divorciada; ignorado/não preenchido); escolaridade das mães em anos de estudo (nenhum; 1 a 7; 8 a 11; 12 ou mais; escolaridade ignorada/não preenchida); ocupação habitual ou ramo de atividade (mães que não trabalham; mães que trabalham; ignorada/não preenchida); zona de residência da mãe (rural; urbana; ignorada/não preenchida); duração da gestação em semanas (22 a 31; 32 a 36; 37 a 41; 42 ou mais; ignorada/não preenchida); tipo de parto (vaginal; cesárea; ignorado/não preenchi- do); número de consultas de pré-natal (nenhuma; 1 a 3; 4 a 6; 7 ou mais; ignorado/não preenchido); sexo (masculino; feminino); raça/cor (não branca: negra, amarela, parda e indígena; branca; ignorada/não preenchida); local de ocorrência do parto (domicílio; hospital; ignorado: quando o parto ocorreu em qualquer outro local ou quando o local de ocorrência do parto foi ignorado ou não preenchido).

Foram considerados como critérios de exclusão: peso do recém-nascido não preenchido; sexo do recém-nascido não preenchido ou ignorado; gravidez múltipla; tipo de gravidez não preenchida.

Os dados foram analisados utilizando-se o pacote estatístico Stata. A associação entre a variável dependente (peso de nascimento, com duas categorias: baixo peso, peso normal) e as demais variáveis (independentes) foi estudada por meio de regressão linear generalizada família Poisson ligação logarítmica com variância robusta, simples e múltipla, que permite a obtenção direta de boa aproximação da razão de prevalências (RP) ${ }^{15,16}$. Para a análise de associação, inicialmente realizou-se o cálculo da regressão simples entre cada uma das variáveis independentes e a variável resposta. Entraram no modelo múltiplo todas as variáveis que na regressão simples apresentaram um valor $\mathrm{p}<0,20$. A seguir, as variáveis que no modelo múltiplo apresentaram $\mathrm{p}>0,10$ foram retiradas gradualmente. Adotou-se nível de significância de 0,10 . A categoria de referência das variáveis, em geral, foi a penúltima, uma vez que a última categoria refere-se a não preenchimento ou situação ignorada. Nas seguintes variáveis utilizou-se outra categoria de referência, para facilitar a compreensão e discussão das proporções de BPN com outros trabalhos: idade da mãe (faixa etária de 20 a 24 anos) e duração da gestação (37 a 41 semanas).

A pesquisa foi aprovada pelo Comitê de Ética da Faculdade de Saúde Pública, de acordo com os requisitos da Resolução 196/96 do Conselho Nacional de Saúde. 


\section{RESULTADOS}

Nos anos de 2006 e 2007, foram preenchidas 3220 declarações de nascidos vivos correspondentes aos filhos de mães residentes em Cruzeiro do Sul e cujos partos ocorreram nesta localidade.

De todas as variáveis do estudo, o peso de nascimento foi a que apresentou maior porcentagem de falta de preenchimento/informa- ção ignorada $(12,36 \%)$, seguida pela raça/cor do recém-nascido $(9,63 \%)$.

Após a aplicação dos critérios de exclusão, restaram 2772 declarações, a partir das quais se realizou a análise dos dados.

O peso de nascimento variou de 560 a 4910 g, com média 3161,5 g e desvio-padrão 535,9 g. A porcentagem de crianças que apresentaram baixo peso ao nascer no período estudado foi 9,13\% (Tabela 1).

Tabela 1: Número e proporção de nascidos vivos, segundo peso ao nascer. Cruzeiro do Sul, Acre, 2006 e 2007.

\begin{tabular}{lcc}
\hline Peso ao nascer (gramas) & $\mathbf{N}$ & $\mathbf{\%}$ \\
\hline$<2500$ & 253 & 9,13 \\
$\geq 2500$ & 2519 & 90,87 \\
\hline Total & $\mathbf{2 7 7 2}$ & $\mathbf{1 0 0}$ \\
\hline
\end{tabular}

Em relação ao local de ocorrência do parto, analisando-se os dados brutos (sem aplicar os critérios de exclusão), observou-se que $85,93 \%$ dos partos ocorreram em ambiente hospitalar, 11,74\% em ambiente domiciliar e $2,33 \%$ em outros locais. Após a aplicação dos critérios de exclusão, verificou-se que $97,4 \%$ das crianças nasceram no hospital e $1,62 \%$ no domicílio. As porcentagens de baixo peso ao nascer (BPN) nos nascidos vivos em ambiente domiciliar e hospitalar foram $26,67 \%$ e $8,74 \%$, respectivamente. Na regressão simples, a razão de prevalências (RP) foi 3,05 com $\mathrm{P}<0,001$.

A idade materna variou de 12 a 47 anos, com média 23,4 anos e desvio padrão 5,9 anos. A proporção de BPN foi maior em nascidos vivos de mães mais jovens, apresentando uma redução em idades intermediárias e aumentando novamente entre filhos de mães com maior idade (Tabela 2).

Houve predominância de mães solteiras $(85,53 \%)$. As outras categorias juntas representaram $13,6 \%$ do total. Os nascidos vivos de mães solteiras foram os que apresentaram maior porcentagem de BPN (9,7\%) (Tabela 2).
Em relação à escolaridade materna, $38,06 \%$ das mães não sabiam ler nem escrever, sendo esta a categoria que apresentou maior proporção de BPN $(10,43 \%)$. A porcentagem de BPN diminui à medida que aumenta a escolaridade materna (Tabela 2).

Quanto à ocupação materna, verificouse que $89,86 \%$ das mães eram "do lar" ou estudantes e $9,42 \%$ referiram trabalhar fora do domicílio. As proporções de BPN entre os nascidos vivos de mães que trabalham fora do domicílio e que não trabalham foram 3,83\% e $9,67 \%$, respectivamente (Tabela 2 ).

Do total de nascidos vivos, $46,79 \%$ eram filhos de mães que residiam em zona urbana. Entre estes, a porcentagem de BPN foi 8,17\%. Entre os nascidos vivos de mães que residiam em zona rural $(53,17 \%)$, a proporção de BPN foi $9,97 \%$ (Tabela 2).

$\mathrm{Na}$ análise de regressão simples entre peso ao nascer e as características relacionadas à mãe, todas as variáveis apresentaram, em pelo menos uma de suas categorias, $\mathrm{p}<0,20$ (Tabela 2). Portanto, todas foram incluídas no modelo múltiplo. 
Tabela 2: Número e proporção de nascidos vivos, segundo as características da mãe e peso; razão de prevalências e P descritivo. Cruzeiro do Sul, Acre, 2006 e 2007.

\begin{tabular}{|c|c|c|c|c|c|c|c|c|}
\hline \multirow{3}{*}{ Variável } & \multirow{3}{*}{ Categorias } & \multicolumn{4}{|c|}{ Peso } & \multirow{3}{*}{ Total } & \multirow{3}{*}{$\mathbf{R P}$} & \multirow{3}{*}{$\mathbf{P}$} \\
\hline & & \multicolumn{2}{|c|}{ Normal } & \multicolumn{2}{|c|}{ Baixo } & & & \\
\hline & & $\mathbf{N}$ & $\%$ & $\mathbf{N}$ & $\%$ & & & \\
\hline \multirow[t]{8}{*}{ Idade } & 12 e 13 & 10 & 83,33 & 2 & 16,67 & 12 & 2,36 & 0,189 \\
\hline & 14 e 15 & 91 & 86,67 & 14 & 13,33 & 105 & 1,89 & 0,020 \\
\hline & 16 e 17 & 254 & 86,6 & 39 & 13,31 & 293 & 1,89 & 0,001 \\
\hline & 18 e 19 & 355 & 89,42 & 42 & 10,58 & 397 & 1,50 & 0,030 \\
\hline & 20 a 24 & 872 & 92,96 & 66 & 7,04 & 938 & 1 & - \\
\hline & 25 a 34 & 798 & 92,04 & 69 & 7,96 & 867 & 1,13 & 0,457 \\
\hline & 35 e mais & 125 & 86,81 & 19 & 13,19 & 144 & 1,87 & 0,010 \\
\hline & Ignorada & 14 & 87,50 & 2 & 12,50 & 16 & 1,77 & 0,393 \\
\hline \multirow{3}{*}{$\begin{array}{l}\text { Estado } \\
\text { Civil }\end{array}$} & Solteira & 2141 & 90,30 & 230 & 9,70 & 2371 & 1,74 & 0,012 \\
\hline & Outros & 356 & 94,43 & 21 & 5,57 & 377 & 1 & - \\
\hline & Ignorado & 22 & 91,67 & 2 & 8,33 & 24 & 1,49 & 0,570 \\
\hline \multirow{5}{*}{$\begin{array}{l}\text { Escolaridade } \\
\text { (em anos de } \\
\text { estudo) }\end{array}$} & Nenhum & 945 & 89,57 & 110 & 10,43 & 1055 & 1,35 & 0,062 \\
\hline & 1 a 7 & 380 & 90,69 & 39 & 9,31 & 419 & 1,20 & 0,355 \\
\hline & 8 a 11 & 563 & 91,54 & 52 & 8,46 & 615 & 1,09 & 0,627 \\
\hline & $\geq 12$ & 622 & 92,28 & 52 & 7,72 & 674 & 1 & - \\
\hline & Igñorada & 9 & 100,00 & 0 & 0,00 & 9 & 0 & 0,000 \\
\hline \multirow[t]{3}{*}{ Ocupação } & trabalha & 2250 & 90,33 & 241 & 9,67 & 2491 & 2,52 & 0,003 \\
\hline & não trabalha & 251 & 96,17 & 10 & 3,83 & 261 & 1 & - \\
\hline & ignorada & 18 & 90,00 & 2 & 10,00 & 20 & 2,61 & 0,194 \\
\hline Zona & Rural & 1327 & 90,03 & 147 & 9,97 & 1474 & 1,22 & 0,101 \\
\hline de & Urbana & 1191 & 91,83 & 106 & 8,17 & 1297 & 1 & - \\
\hline residência & Ignorada & 1 & 100,00 & 0 & 0,00 & 1 & 0 & 0,000 \\
\hline
\end{tabular}

Quanto à duração da gestação, 2,64\% dos nascidos vivos foram prematuros. A porcentagem de BPN aumenta à medida que diminui a duração da gestação (Tabela 3).

Do total de partos, $24,86 \%$ foram por operação cesariana. As proporções de BPN entre os nascidos vivos de parto vaginal e cesárea foram $9,55 \%$ e $7,98 \%$, respectivamente (Tabela 3).

Do total de mães, 4,73\% não realizaram nenhuma consulta de pré-natal. A maior porcentagem de BPN ocorreu nos nascidos vivos de mães que realizaram de 1 a 3 consultas $(18,13 \%)$ (Tabela 3$)$.

Dentre as características relacionadas à gestação e ao parto, no cálculo da regressão simples (Tabela 3), a variável tipo de parto apresentou $R P=1,19$ com $P=0,220$ (parto va- ginal em relação à cesárea), portanto, não entrou no modelo múltiplo.

Do total de nascidos vivos, $51,3 \%$ foram do sexo masculino e $48,7 \%$ do sexo feminino. O sexo feminino apresentou maior porcentagem de BPN $(10,44 \%)$ (Tabela 4).

Houve predominância de crianças da raça/cor parda $(68,66 \%)$, seguida por branca $(30,68 \%)$, indígena $(0,59 \%)$ e negra $(0,07 \%)$. Não houve nascimento de crianças da raça/ cor amarela. Nos nascidos vivos de raça/cor branca, a porcentagem de BPN foi 7,38\%. Entre os de outras raças/cores foi 9,79\% (Tabela 4).

A análise de regressão simples das variáveis sexo e raça/cor do recém-nascido pode ser observada na tabela 4 . As duas variáveis entraram no modelo múltiplo. 
Tabela 3: Número e proporção de nascidos vivos, segundo as características da gestação e do parto e peso; razão de prevalências e P descritivo. Cruzeiro do Sul, Acre, 2006 e 2007.

\begin{tabular}{|c|c|c|c|c|c|c|c|c|}
\hline \multirow{3}{*}{ Variável } & \multirow{3}{*}{ Categorias } & \multicolumn{4}{|c|}{ Peso } & \multirow{3}{*}{ Total } & \multirow{3}{*}{$\mathbf{R P}$} & \multirow{3}{*}{$\mathbf{P}$} \\
\hline & & \multicolumn{2}{|c|}{ Normal } & \multicolumn{2}{|c|}{ Baixo } & & & \\
\hline & & $\mathbf{N}$ & $\%$ & $\mathbf{N}$ & $\%$ & & & \\
\hline \multirow{5}{*}{$\begin{array}{l}\text { Duração da } \\
\text { gestação }\end{array}$} & De 22 a 31 & 2 & 12,50 & 14 & 87,50 & 16 & 12,18 & 0,000 \\
\hline & De 32 a 36 & 13 & 22,81 & 44 & 77,19 & 57 & 10,74 & 0,000 \\
\hline & De 37 a 41 & 2481 & 92,82 & 192 & 7,18 & 2673 & 1 & - \\
\hline & $\geq 42$ & 11 & 91,67 & 1 & 8,33 & 12 & 1,16 & 0,877 \\
\hline & Ign̄orada & 12 & 85,71 & 2 & 14,29 & 14 & 1,98 & 0,296 \\
\hline \multirow{3}{*}{$\begin{array}{l}\text { Tipo de } \\
\text { parto }\end{array}$} & Vaginal & 1876 & 90,45 & 198 & 9,55 & 2074 & 1,19 & 0,220 \\
\hline & Cesáreo & 634 & 92,02 & 55 & 7,98 & 689 & 1 & - \\
\hline & Ignorado & 9 & 100,00 & 0 & 0,00 & 9 & 0 & 0,000 \\
\hline $\mathrm{N}^{o} \mathrm{de}$ & Nenhuma & 113 & 86,26 & 18 & 13,74 & 131 & 1,82 & 0,009 \\
\hline consultas & 1 a 3 & 149 & 81,87 & 33 & 18,13 & 182 & 2,41 & 0,000 \\
\hline de pré- & 4 a 6 & 279 & 86,92 & 42 & 13,08 & 321 & 1,73 & 0,001 \\
\hline \multirow[t]{2}{*}{ natal } & 7 e mais & 1943 & 92,48 & 158 & 7,52 & 2101 & 1 & - \\
\hline & Ignorado & 35 & 94,59 & 2 & 5,41 & 37 & 0,71 & 0,633 \\
\hline
\end{tabular}

Tabela 4: Número e proporção de nascidos vivos, segundo as características do recém-nascido e peso; razão de prevalências e P descritivo. Cruzeiro do Sul, Acre, 2006 e 2007.

\begin{tabular}{ccccccccc}
\hline \multirow{2}{*}{ Variável } & \multirow{8}{*}{ Categorias } & \multicolumn{2}{c}{ Normal } & Peso & \multirow{2}{*}{ Baixo } & Total & \multirow{2}{*}{ RP } & \multirow{2}{*}{$\mathbf{P}$} \\
& & $\mathbf{N}$ & $\mathbf{\%}$ & $\mathbf{N}$ & $\mathbf{\%}$ & & & \\
\hline Sexo & Masculino & 1310 & 92,12 & 112 & 7,88 & 1422 & 1 & - \\
& Feminino & 1209 & 89,56 & 141 & 10,44 & 1350 & 1,32 & 0,019 \\
& & & & & & & & \\
Raça/cor & Não branca & 1686 & 90,21 & 183 & 9,79 & 1869 & 1,32 & 0,046 \\
& Branca & 766 & 92,62 & 61 & 7,38 & 827 & 1 & - \\
& Ignorada & 67 & 88,16 & 9 & 11,84 & 76 & 1,60 & 0,159 \\
\hline
\end{tabular}

Apenas a variável tipo de parto não entrou no modelo múltiplo. Entre as demais variáveis, não permaneceram no modelo final (pois nesta etapa apresentaram $\mathrm{P}$ maior que $0,10)$ as seguintes: escolaridade e zona de residência materna. Na tabela 5 visualiza-se o modelo final com as variáveis cujos $P$ descritivos foram menor que 0,10 .

\section{DISCUSSÃO}

A proporção de baixo peso ao nascer foi $9,13 \%$, uma taxa maior do que a apresentada no município de Rio Branco (AC) e no Brasil que, em 2005, foram respectivamente $8,2 \% \mathrm{e}$ $8,1 \%{ }^{13}$. Na área da saúde pública, o peso de nascimento pode ser considerado um importante indicador, pois reflete as condições de vida da mulher bem como a qualidade da assistência recebida durante a gravidez ${ }^{17}$. O monitoramento da prevalência dessa condição é um instrumento importante na área maternoinfantil uma vez que as sequelas decorrentes do nascimento de crianças com peso não adequado e a necessidade de um maior acompanhamento e cuidado a esses indivíduos são evidenciados de forma consistente na literatu$\mathrm{ra}^{3-4,18-20}$.

A ocorrência de partos no hospital $(85,93 \%)$ foi menor que a encontrada em outras localidades ${ }^{1}$. Segundo o Instituto Brasileiro de Geografia e Estatística, em 2000, 42,21\% da população do Cruzeiro do Sul tinha sua resi- 
Tabela 5: Razões de prevalências (RP) de baixo peso ao nascer e respectivos intervalos com $90 \%$ de confiança (IC) e P descritivos. Cruzeiro do Sul, Acre, 2006 e 2007. Modelo final.

\begin{tabular}{|c|c|c|c|c|c|}
\hline & & & & & \\
\hline Variável & Categorias & Bruto & Ajustado & IC $90 \%$ & $\mathbf{P}$ \\
\hline Duração & 22 a 31 & 12,18 & 11,32 & $8,51-15,0$ & 0,000 \\
\hline & 32 a 36 & 10,74 & 10,01 & $58,26-12,13$ & 0,000 \\
\hline gestação & 37 a 41 & 1 & 1 & - & - \\
\hline (semanas) & 42 e mais & 1,16 & 1,25 & $0,22-6,93$ & 0,828 \\
\hline & Ignorada & 1,98 & 2,26 & $0,74-6,83$ & 0,224 \\
\hline Local de & Domicílio & 3,05 & 3,13 & $1,92-5,09$ & 0,000 \\
\hline ocorrência do & Hospital & 1 & 1 & & - \\
\hline parto & Ignorado & 2,11 & 1,80 & $0,97-3,31$ & 0,112 \\
\hline Consultas de & Nenhuma & 1,82 & 1,02 & $0,68-1,52$ & 0,914 \\
\hline pré-natal & 1 a 3 & 2,41 & 1,42 & $1,06-1,90$ & 0,049 \\
\hline & 4 a 6 & 1,73 & 1,15 & $0,90-1,47$ & 0,326 \\
\hline & 7 ou mais & 1 & 1 & & - \\
\hline & Ignorado & 0,71 & 0,76 & $0,27-2,12$ & 0,669 \\
\hline Idade da mãe & 12 a 13 & 2,36 & 2,97 & $1,01-8,79$ & 0,099 \\
\hline (anos) & 14 a 15 & 1,89 & 1,32 & $0,86-2,04$ & 0,283 \\
\hline & 16 a 17 & 1,89 & 1,49 & $1,10-2,03$ & 0,030 \\
\hline & 18 a 19 & 1,50 & 1,33 & $1,01-1,77$ & 0,091 \\
\hline & 20 a 24 & 1 & 1 & - & - \\
\hline & 25 a 34 & 1,13 & 1,09 & $0,85-1,41$ & 0,536 \\
\hline & 35 ou mais & 1,87 & 1,78 & $1,20-2,63$ & 0,014 \\
\hline & Ignorada & 1,77 & 2,32 & $0,79-6,80$ & 0,195 \\
\hline & & & & $1,06-2,14$ & 0,052 \\
\hline Estado civil & Solteira & 1,74 & 1,51 & & \\
\hline & Outros & 1 & 1 & - & - \\
\hline & Ignorado & 1,49 & 1,04 & $0,39-2,77$ & 0,942 \\
\hline Ocupação da & Não trabalha & 2,52 & 1,92 & & \\
\hline mãe & Trabalha & 1 & 1 & & - \\
\hline & Ignorada & 2,61 & 3,36 & $0,99-11,44$ & 0,103 \\
\hline & & & & $1,04-1,60$ & 0,044 \\
\hline Raça/cor do & Não branca & 1,32 & 1,29 & & \\
\hline recém-nascido & Branca & 1 & 1 & - & - \\
\hline & Ignorada & 1,60 & 1,34 & $0,86-2,08$ & 0,272 \\
\hline Sexo do & Masculino & 1 & 1 & - & - \\
\hline recém-nascido & Feminino & 1,32 & 1,30 & $1,08-1,56$ & 0,018 \\
\hline
\end{tabular}

dência localizada em área rural ${ }^{21}$, o que pode explicar o elevado número de partos no domicílio. A proporção de BPN foi 3,13 vezes maior entre as crianças que nasceram no domicílio quando comparadas às que nasceram no hospital. Esse achado reforça a importância da cobertura prénatal e do acesso aos serviços de saúde.

Os nascidos vivos de mães entre $14 \mathrm{e}$ 15 anos apresentaram elevada porcentagem de
BPN com razão de prevalência 1,32 , porém estatisticamente não significante. Associação estatisticamente significante foi detectada entre o BPN e as seguintes faixas etárias da mãe: 12 e 13 anos, 16 e 17 anos, 18 e 19 anos, 35 anos e mais. Esse resultado corrobora com outros que relacionam a gravidez nos extremos da vida reprodutiva a um maior risco de nascimento de crianças com baixo peso ${ }^{22-24}$. No en- 
tanto, a associação entre essas variáveis não foi encontrada em estudo realizado em São Paulo. Segundo os autores, a adequada assistência pré-natal possivelmente foi responsável por diminuir a frequência de baixo peso ao nascer em filhos de mães adolescentes ${ }^{25}$.

Em relação ao estado civil, verificou-se associação estatisticamente significante entre o BPN e filhos de mães solteiras. A associação entre essas variáveis pode estar relacionada, entre outros motivos, à presença do companheiro no domicílio, pois alguns trabalhos mostraram que a ausência do pai está associada à falta de adesão ao pré-natal e ao $\mathrm{BPN}^{26,27}$.

$\mathrm{Na}$ análise múltipla, não foi detectada associação estatisticamente significante entre escolaridade e BPN, concordando com os resultados do estudo de Nascimento ${ }^{24}$. No Maranhão ${ }^{28}$, a proporção de mães com 8 a 11 anos de estudo $(75,1 \%)$ foi maior que a detectada no presente estudo.

Em relação à ocupação materna, houve um aumento estatisticamente significante da prevalência de BPN em crianças cujas mães não trabalhavam. A proteção contra o BPN, conferida pelo trabalho materno fora do lar, possivelmente está ligada ao fator socioeconômico. Monteiro e Freitas ${ }^{29}$ apontam que o acesso a alimentação e aos serviços essenciais, bem como a qualidade do ambiente são fatores que dependem do poder aquisitivo.

A porcentagem de partos prematuros $(2,64 \%)$ foi menor que a média brasileira $(6,6 \%)$ e a média do Estado do Acre $(3,3 \%)$, em $2005^{13}$. A prevalência de BPN se mostrou elevada quando a duração da gestação era de 22 a 31 semanas e de 32 a 36 semanas. Em outro estudo, as crianças prematuras nascidas entre 21 e 27 semanas de gestação apresentaram um risco 15,6 vezes maior de nascer com baixo peso que crianças nascidas a termo ${ }^{30}$. O nascimento de crianças prematuras nem sempre pode ser evitado, porém pode-se atuar com foco em alguns fatores de risco para a prematuridade. A adequada assistência durante a gra- videz é de importância fundamental pois possibilita a detecção e o tratamento de hábitos e condições maternas que podem contribuir para a ocorrência de partos prematuros.

O resultado da análise de associação entre o BPN e o tipo de parto não foi estatisticamente significante. Outros autores também não encontraram qualquer associação entre essas variáveis ${ }^{30,31}$.

Em relação ao número de consultas no pré-natal, observou-se um aumento estatisticamente significante da prevalência de BPN entre os nascidos vivos cujas mães realizaram de 1 a 3 consultas. Ao contrário do esperado, a categoria nenhuma consulta não foi significante. Esse fato alerta para a possibilidade de má qualidade dessa informação, sendo necessária maior atenção no momento da obtenção e registro dessa variável. Pesquisas confirmam que a assistência pré-natal está se tornando mais acessível nas diversas regiões do Brasil ${ }^{13}$. Por sua relevância, esse serviço deve ser alvo de avaliações periódicas com o intuito de garantir a acessibilidade e a qualidade da assistência tornando possível, dessa forma, uma oportuna atuação sobre os fatores associados ao BPN e outras condições indesejáveis.

A associação entre o BPN e o sexo feminino foi estatisticamente significante. As meninas apresentaram uma proporção de BPN 1,3 vezes maior quando comparadas aos meninos. Outros estudos também detectaram essa associação $0^{28,29}$. A relação entre essas variáveis era esperada dado que para a mesma idade gestacional, as meninas apresentam menor peso que os meninos?.

Em relação à raça/cor do nascido vivo, identificou-se associação estatisticamente significativa entre o BPN e os não-brancos. A proporção de BPN nos não brancos foi $29 \%$ maior que a proporção nos brancos. O conhecimento desta característica na população é importante devido à existência de desigualdades sociais e em consequência da ocorrência de morbidades 
relacionada à etnia $/ \operatorname{cor}^{30}$. Esses fatores podem conferir diferentes graus de vulnerabilidade dos indivíduos a determinadas doenças.

A proporção de baixo peso ao nascer foi $9,13 \%$. Os fatores associados foram: prematuridade; nascimento no domicílio; sexo feminino; idade materna entre 12 e 13 anos, 16 e 17 anos, 18 e 19 anos, 35 e mais anos; realização de 1 a 3 consultas de pré-natal e possivelmente nenhuma, crianças não brancas, mães sem ocupação fora do lar e mães solteiras.

\section{REFERÊNCIAS}

1. Orlonski S, Dellagrana RA, Rech CR, Araújo EDS. Estado nutricional e fatores associados ao déficit de estatura em crianças atendidas por uma unidade de ensino básico de tempo integral. Rev. bras. crescimento desenvolv. hum. 2009; 19(1): 54-62.

2. ACC/SCN. Low Birthweight: Report of a Meeting in Dhaka, Bangladesh on 14-17 June 1999. Eds. Pojda J and Kelley L. Nutrition Policy Paper. 2000; 18.

3. Sarni ROS, Souza FIS, Pitta TS, Fernandez AP, Hix S, Fonseca FA. Baixo peso ao nascer: influência na pressão arterial, composição corporal e antropometria. Arq Med ABC. 2005; 30(2):76-82.

4. Barroso GS, Sichieri R, Salles-Costa R. Fatores associados ao déficit nutricional em crianças residentes em uma área de prevalência elevada de insegurança alimentar. Rev Bras Epidemiol. 2008; 11(3): 484-94.

5. Organização Mundial da Saúde. Classificação Internacional das Doenças, $10^{\circ}$ revisão II, 1993.

6. Camiña RS. Prevalência de baixo peso ao nascer e fatores associados nos municípios da $7^{\mathrm{a}}$ regional de saúde de Joaçaba, Santa Catarina, no período de 1995-1999 [dissertação]. Santa Catarina: Universidade do Oeste de Santa Catarina; 2005 [acesso em 24 mar 2007] Disponível em: www.unoescjba.edu.br/cursos/mestrado/ msaude/dissertacoes_defendidas.php 157k.htm
A etiologia multifatorial do BPN dificulta o estabelecimento de um programa que possa resolver esse problema. Ações para a redução desse agravo extrapolam o setor saúde e exigem um esforço organizado de vários setores da sociedade. No campo específico da saúde, a realização de intervenções simples e programas rotineiros de assistência à saúde, desde que sejam efetivos e de qualidade, podem contribuir para a redução do problema e de suas consequências.

7. Federação Brasileira das Sociedades de Ginecologia e Obstetrícia. Tratado de Obstetrícia. Rio de Janeiro: Revinter; 2000.

8. Bezerra CB, Oliveira SMJV, Latorre MRDO. Prevalência e fatores associados à prematuridade entre gestantes submetidas à inibição de trabalho de parto prematuro. Rev Bras Saúde Mater Infant. 2006; 6(2): 223-229.

9. Silva AMR. Fatores de risco para nascimentos pré-termo no Município de Londrina - Paraná [tese] São Paulo: Faculdade de Saúde Pública da Universidade de São Paulo; 2008.

10. Cascaes AM, Gauche H, Baramarchi FM, Borges CM, Peres KG. Prematuridade e fatores associados no Estado de Santa Catarina, Brasil, no ano de 2005: análise dos dados do Sistema de Informações sobre Nascidos Vivos. Cad Saúde Pública. 2008; 24(5): 1024-1032.

11. United Nations Children's Fund and World Health Organization. Low Birthweight: Country, regional and global estimates. New York: UNICEF; 2004.

12. United Nations Children's Fund. Situação mundial da infância 2008. Brasília: MS; 2008.

13. BRASIL. Ministério da Saúde. Departamento de Informação e informática do SUS. Cadernos de Informações de Saúde [acesso em 15 jan 2009]. Disponível em: http://www.datasus.gov.br

14. BRASIL. Fundação Nacional de Saúde. Manual de instruções para o preenchimento da declaração de nascido vivo. 3.ed. Brasília: MS, 2001. 
15. Barros AJD, Hirakata VN. Alternatives for logistic regression in cross-sectional studies: an empirical comparison of models that directly estimate the prevalence ratio. BMC Medical Research Methodology. 2003; 3(21).

16. Zou G. A modified Poisson Regression Approach to Prospective Studies with Binary Data. Am J epidemiol. 2004; 159(7):702-706.

17. Trevisan MR, De Lorenzi DRS, Araújo NM, Ésber K. Perfil da assistência prénatal entre usuários do Sistema. Único de Saúde em Caxias do Sul. Rev Bras Ginecol Obstet. 2002; 24(5): 293-299.

18. Yamamoto RM, Schoeps DO, Abreu LC, Leone C. Peso insuficiente ao nascer e crescimento alcançado na idade pré-escolar, por crianças atendidas em creches filantrópicas do município de Santo André, São Paulo, Brasil. Rev. Bras. Saude Mater. Infant. 2009; 9(4): 477-485.

19. Oliveira AG, Siqueira PP, Abreu LC. Cuidados nutricionais no recém-nascido de muito baixo peso. Rev. bras. crescimento desenvolv. hum. 2008; 18(2):148-154.

20. Bismarck-Nasr EM, Frutuoso MFP, Gamabardella AMD. Efeitos tardios do baixo peso ao nascer. Rev. bras. crescimento desenvolv. hum. 2008; 18(1): 98-103.

21. Instituto Brasileiro de Geografia e Estatística. Cidades, Acre, Cruzeiro do Sul [acesso em 08 fev 2009]. Disponível em: http://www.ibge.gov.br/cidadesat/ topwindow.htm? 1

22. Nascimento LFC, Gotlieb SLD. Fatores de risco para o baixo peso ao nascer, com base em informações da Declaração de Nascido Vivo em Guaratinguetá, SP, no ano de 1998. Inf Epidemiol SUS. 2001; 10(3): 113-120.

23. Aquino-Cunha M. Gestação na adolescência: relação com o baixo peso ao nascer. Rev Bras Ginecol Obstet. 2002; 24(8): 513-519.

24. Nascimento LFC. Estudo transversal sobre fatores associados ao baixo peso ao nascer a partir de informações obtidas em sala de vacinação. Rev Bras Saude Mater Infant. 2003; 3(1): 37-42.

25. Suzuki CM, Ceccon MEJ, Falcão MC, Vaz FAC. Análise comparativa da freqüência de prematuridade e baixo peso entre filhos de mães adolescentes e adultas. Rev. bras. crescimento desenvolv. hum. 2007; 17(3): 95-103.

26. Coimbra LC, Silva AAM, Mochel EG, Alves MTSSB, Ribeiro VS, Aragão VMF et al. Fatores associados à inadequação do uso da assistência pré-natal. Rev Saúde Pública. 2003; 37(4): 456-62.

27. Minasava R, Barbosa MA, Malagoni L, Andraus LMS. Fatores associados ao baixo peso ao nascer no Estado de Goiás. Rev Eletrônica Enferm. 2004 [acesso em 24 mar 2004]; 6(3). Disponível em: http:// www.fen.ufg.br

28. Santos GHN, Martins MG, Sousa MS. Gravidez na adolescência e fatores associados com baixo peso ao nascer. Rev Bras Ginecol Obstet. 2008; 30(5): 224-31.

29. Monteiro CA, Freitas ICM. Evolução de condicionantes socioeconômicas da saúde na infância na cidade de São Paulo (1984-1996). Rev Saúde Pública. 2000; 34(6S): 8-12.

30. Guimarães EAA, Velásquez-Neléndez G. Determinantes do baixo peso ao nascer a partir do Sistema de Informação Sobre Nascidos Vivos em Itaúna, Minas Gerais. Rev Bras Saúde Mater Infant 2002; 2(3): 283-290.

31. Stein-Backes MT, Flores-Soares MC. Las enfermedades intercurrentes dutante la gestación y SUS consecuencias sobre el peso del recién nacido. Rev Colomb Obstet Ginecol. 2008; 59(2): 103-110.

32. Carniel EF, Zanolli ML, Antônio MARGM, Morcillo AM. Determinantes do baixo peso ao nascer a partir das declarações de nascidos vivos. Rev Bras Epidemiol. 2008; 11(1): 169-79.

33. Zaganelli FL. Aspectos do perfil social da gestação e do parto da adolescente e da mulher adulta e suas repercussões sobre o recém-nascido [dissertação]. Belo Horizonte: Faculdade de Medicina da UFMG; 2006.

34. Carniel EF. Caracterização dos recém-nascidos e de suas mães, a partir das declarações de nascidos vivos de Campinas (SP), no ano de 2001 [dissertação]. São Paulo: Faculdade de Ciências Médicas da Universidade Estadual de Campinas; 2005.

Recebido em 12/03/10

Modificado em 16/08/10 Aceito em 09/09/10 